TRANSACTIONS OF THE

AMERICAN MATHEMATICAL SOCIETY

Volume 358, Number 8, Pages 3285-3303

S 0002-9947(06)04122-5

Article electronically published on March 24, 2006

\title{
PRIME GEODESIC THEOREM FOR HIGHER-DIMENSIONAL HYPERBOLIC MANIFOLD
}

\author{
MAKI NAKASUJI
}

\begin{abstract}
For a $(d+1)$-dimensional hyperbolic manifold $\mathcal{M}$, we consider an estimate of the error term of the prime geodesic theorem. Put the fundamental group $\Gamma$ of $\mathcal{M}$ to be a discrete subgroup of $S O_{e}(d+1,1)$ with cofinite volume. When the contribution of the discrete spectrum of the Laplace-Beltrami operator is larger than that of the continuous spectrum in Weyl's law, we obtained a lower estimate $\Omega_{ \pm}\left(\frac{x^{d / 2}(\log \log x)^{1 /(d+1)}}{\log x}\right)$ as $x$ goes to $\infty$.
\end{abstract}

\section{INTRODUCTION}

In this paper we will study the asymptotic behavior of a family of counting functions connected with the fundamental group $\Gamma$ of a $(d+1)$-dimensional hyperbolic manifold $\mathcal{M}$ of finite volume.

If $\gamma \in \Gamma$, then for each free homotopy class of $\gamma$ there exists one closed geodesic on $\mathcal{M}$. We will denote its length by $l(\gamma)$ and define its norm by $N(\gamma)=e^{l(\gamma)}$. When we put $\pi_{\Gamma}(x)$ to be the number of primitive elements $\gamma \in \Gamma$ such that $N(\gamma) \leq x$, the asymptotic formula is

$$
\pi_{\Gamma}(x)=\operatorname{li}\left(x^{d}\right)+\sum_{n=1}^{M} \operatorname{li}\left(x^{s_{n}}\right)+(\text { error }),
$$

where $s_{1}, \cdots, s_{M}$ are the zeros of the Selberg zeta function $Z_{\Gamma}(s)$ in the interval $\left(\frac{d}{2}, d\right)$. We call this formula a prime geodesic theorem. The chief concern of this paper is to give a lower estimate of the error term in (1.1).

In the previous paper [7, we obtain a lower bound in 3-dimensional cases, i.e. $d=2$, by considering the contribution of the spectra of the Laplacian.

Theorem 1.1 ([7, Theorem 1.4]). Let $\Gamma \subset P S L(2, \mathbf{C})$ be a cofinite subgroup. We assume

$$
N_{\Gamma}(T) \sim \frac{\operatorname{vol}\left(\Gamma \backslash \mathbf{H}^{3}\right)}{6 \pi^{2}} T^{3},
$$

where $\mathbf{H}^{3}$ is a 3-dimensional hyperbolic space and

$$
N_{\Gamma}(T):=\sharp\left\{\lambda_{n} \mid \lambda_{n}<1+T^{2}\right\}
$$

Received by the editors April 22, 2003.

2000 Mathematics Subject Classification. Primary 11M36, 11F72.

Key words and phrases. Prime geodesic theorem, Selberg zeta function, Weyl's law.

(C)2006 American Mathematical Society Reverts to public domain 28 years from publication 
is the counting function with multiplicity for $\lambda_{n}$ being the eigenvalues of the Laplacian on $L^{2}\left(\Gamma \backslash \mathbf{H}^{3}\right)$. Then we have

$$
\pi_{\Gamma}(x)=\operatorname{li}\left(x^{2}\right)+\sum_{n=1}^{M} \operatorname{li}\left(x^{s_{n}}\right)+\Omega_{ \pm}\left(\frac{x(\log \log x)^{\frac{1}{3}}}{\log x}\right) \quad \text { as } x \rightarrow \infty .
$$

A generalization of Weyl's law for a 3-dimensional hyperbolic manifold is known as

$$
N_{\Gamma}(T)-\frac{1}{4 \pi} \int_{-T}^{T} \frac{\varphi^{\prime}}{\varphi}(1+i t) d t \sim \frac{\operatorname{vol}\left(\Gamma \backslash \mathbf{H}^{3}\right)}{6 \pi^{2}} T^{3},
$$

where $\varphi(s)$ is the scattering determinant.

Compared with that $N_{\Gamma}(T)$ expresses the contribution of the discrete spectra, the second term in (1.3) expresses that of the continuous one. The assumption (1.2) means $N_{\Gamma}(T)$ is the main term in (1.3).

Here we would like to extend the estimate in Theorem 1.1 to $\Gamma$ being higherdimensional discrete subgroups in a connected noncompact semisimple Lie group $S O_{e}(d+1,1)$. Let $S O_{e}(d+1,1)=K A_{\mathfrak{p}} N$ be the Iwasawa decomposition and let $u=u(h)\left(h \in A_{\mathfrak{p}}\right)$ be a coordinate function on $A_{\mathfrak{p}}$ (see Section 2). In fact, for $\gamma \in \Gamma$ the number $u_{\gamma}=u(h(\gamma))$ is essentially the length of the minimal geodesic in the homotopy class corresponding to $\gamma$. Hence, here we put the norm for $\gamma$ as $N(\gamma):=e^{u(h(\gamma))}$. Then our main theorem is as follows.

Theorem 1.2. Let $\Gamma \subset S O_{e}(d+1,1)$ be a discrete subgroup with cofinite volume. When

$$
-\int_{-T}^{T} \frac{\varphi^{\prime}}{\varphi}\left(\frac{d}{2}+i t\right) d t=o\left(T^{d+1}\right)
$$

for the scattering determinant $\varphi$, we then have

$$
\pi_{\Gamma}(x)=\operatorname{li}\left(x^{d}\right)+\sum_{n=1}^{M} \operatorname{li}\left(x^{s_{n}}\right)+\Omega_{ \pm}\left(\frac{x^{\frac{d}{2}}(\log \log x)^{\frac{1}{d+1}}}{\log x}\right)
$$

as $x \longrightarrow \infty$.

For the proof of this theorem, we need the Selberg trace formula for our $\Gamma$. Gangolli-Warner 2] obtained the formula for noncompact simple Lie groups of rank one with finite center. In Section 2, we will mention the Selberg trace formula and Selberg zeta function for the case of $S O_{e}(d+1,1)$. In Section 3, we will show some properties for this zeta function. In the last section we will prove the main theorem by applying the propositions in Section 3 .

\section{Preliminaries}

We will begin with the general theory of semisimple Lie groups. Let $G$ be a connected noncompact simple Lie group with finite center, and let $K$ be the maximal compact subgroup. Denote their respective Lie algebras by $\mathfrak{g}$ and $\mathfrak{k}$. Let the Cartan decomposition of $\mathfrak{g}$ with respect to the involution $\theta$ be $\mathfrak{g}=\mathfrak{k}+\mathfrak{p}$. Let $\mathfrak{a}_{\mathfrak{p}}$ be a maximal abelian subspace of $\mathfrak{p}$. We assume $\operatorname{dim} \mathfrak{a}_{\mathfrak{p}}=1$.

Extend $\mathfrak{a}_{\mathfrak{p}}$ to a maximal abelian $\theta$-stable subalgebra $\mathfrak{a}$ of $\mathfrak{g}$, so that $\mathfrak{a}=\mathfrak{a}_{\mathfrak{k}}+\mathfrak{a}_{\mathfrak{p}}$ with $\mathfrak{a}_{\mathfrak{k}}=\mathfrak{a} \cap \mathfrak{k}, \mathfrak{a}_{\mathfrak{p}}=\mathfrak{a} \cap \mathfrak{p}$.

We denote by $\mathfrak{g}^{\mathbf{C}}, \mathfrak{k}^{\mathbf{C}}, \mathfrak{a}^{\mathbf{C}}$ the complexification of $\mathfrak{g}, \mathfrak{k}$ and $\mathfrak{a}$, respectively. Then $\mathfrak{a}^{\mathbf{C}}$ is a Cartan subalgebra of $\mathfrak{g}^{\mathbf{C}}$. Let $\Phi=\Phi\left(\mathfrak{g}^{\mathbf{C}}, \mathfrak{a}^{\mathbf{C}}\right)$ be the set of roots of $\left(\mathfrak{g}^{\mathbf{C}}, \mathfrak{a}^{\mathbf{C}}\right)$. 
Order the dual spaces of $\mathfrak{a}_{\mathfrak{p}}$ and $\mathfrak{a}_{\mathfrak{p}}+i \mathfrak{a}_{\mathfrak{k}}$ compatibly, and let $\Phi^{+}$be the set of positive roots under this order. Let $P_{+}=\left\{\alpha \in \Phi^{+} \mid \alpha \not \equiv 0\right.$ on $\left.\mathfrak{a}_{\mathfrak{p}}\right\}, P_{-}=\{\alpha \in$ $\Phi^{+} \mid \alpha \equiv 0$ on $\left.\mathfrak{a}_{\mathfrak{p}}\right\}$ and put $\rho=\frac{1}{2} \sum_{\alpha \in P_{+}} \alpha$.

Let $\mathfrak{g}=\mathfrak{k}+\mathfrak{a}_{\mathfrak{p}}+\mathfrak{n}$ and $G=K A_{\mathfrak{p}} N$ be the Iwasawa decompositions corresponding to these orders $\left(A_{\mathfrak{p}}=\exp \mathfrak{a}_{\mathfrak{p}}, N=\exp \mathfrak{n}\right)$.

Put $\Sigma$ to be the set of the elements of $P_{+}$restricted to $\mathfrak{a}_{\mathfrak{p}}$. Then $\Sigma=\{\beta\}$ or $\{\beta, 2 \beta\}$. Let $p$ (resp. $q$ ) be the number of roots in $P_{+}$whose restriction to $\mathfrak{a}_{\mathfrak{p}}$ is $\beta$ (resp. $2 \beta$ ). Choose $H_{0} \in \mathfrak{a}_{\mathfrak{p}}$ such that $\beta\left(H_{0}\right)=1$. We denote by $\rho_{0}$ the number $\rho\left(H_{0}\right)$.

Throughout this paper we put $G$ to be the connected noncompact semisimple Lie group $S O_{e}(d+1,1)$. In our situation, we have

$$
\mathfrak{a}=\mathfrak{a}_{\mathfrak{p}}=\left\{\left(\begin{array}{ccc}
0 & & \\
& 0 & a \\
& a & 0
\end{array}\right) \mid 0: d \times d \text { matrix, } a \in \mathbf{R}\right\},
$$

$p=d, q=0$ and $\rho_{0}=\frac{d}{2}$.

Let

$$
K=\left\{g \in\left(\begin{array}{cc}
g_{0} & 0 \\
t_{0} & 1
\end{array}\right) \mid 0 \in \mathbf{R}^{d+1}, g_{0} \in S O(d)\right\} \cong S O(d)
$$

then $K$ is a maximal compact subgroup of $G$.

Let $\Gamma$ be a cofinite subgroup of $G$ and let $\gamma \in \Gamma$ be a semisimple element which is not elliptic, i.e. which is not conjugate to any element of $K$. Then $\gamma$ is conjugate to some element of $A$, say $h(\gamma)$. Let $h(\gamma)=h_{\mathfrak{k}}(\gamma) h_{\mathfrak{p}}(\gamma)$, with $h_{\mathfrak{k}}(\gamma) \in A_{\mathfrak{k}}, h_{\mathfrak{p}}(\gamma) \in A_{\mathfrak{p}}$. For $h_{\mathfrak{p}} \in A_{\mathfrak{p}}$ put $u\left(h_{\mathfrak{p}}(\gamma)\right)=\beta\left(\log h_{\mathfrak{p}}(\gamma)\right)$. Then $u(h)$ is a coordinate function on $A_{\mathfrak{p}}$ and the norm of $\gamma$ is defined as

$$
N(\gamma)=e^{u(h(\gamma))}=\exp (\beta(\log h(\gamma))) .
$$

An element $\gamma \in \Gamma-\{1\}$ is called primitive if and only if it is not an essential power of any other element. A conjugacy class $\{\gamma\}$ in $\Gamma$ is called primitive if each $\gamma$ in the class has this property.

We choose and fix $\varepsilon_{0}>0$ which is smaller than all of the numbers $u(h(\gamma))$ for $\gamma \in \Gamma$, and let $g$ be a real-valued function in $C^{\infty}$ such that

(i) $g$ is even,

(ii) $g$ vanishes in the neighborhood of 0 ,

(iii) for $|x| \geq \varepsilon_{0}, g$ is a constant of some positive integer, equal to $\kappa$, especially $\kappa=d^{\frac{d+1}{2}}$ if $d$ is odd,

(iv) $0 \leq g \leq \kappa$.

Gangolli-Warner obtained the trace formula for some noncompact quotients of symmetric spaces of rank 1 [2, Theorem 1.2]. Applying our case $G=S O_{e}(d+1,1)$ to their formula, we have the following.

Proposition 2.1. Let $G=S O_{e}(d+1,1)$ and $K$ be as in (2.1). Let $\mathcal{C}^{p}(K \backslash G / K)$ be a Fréchet space as in [2, p. 4]. For $f_{s} \in \mathcal{C}^{p}(K \backslash G / K)$ with $p<1$, let $\hat{f}_{s}$ be the 
spherical Fourier transform. Then we have

$$
\begin{aligned}
\sum_{n=0}^{\infty} \hat{f}_{s}\left(r_{n}\right)= & {[Z(\Gamma)] \operatorname{vol}(G / \Gamma) f_{s}(1) } \\
& +\sum_{\{\gamma\}} N(\gamma)^{\frac{d}{2}} \log N\left(\gamma_{0}\right) C(h(\gamma)) g(\log N(\gamma)) N(\gamma)^{-s} \\
& +\frac{1}{4 \pi} \int_{-\infty}^{\infty} \hat{f}_{s}(r) \frac{\varphi^{\prime}}{\varphi}\left(\frac{d}{2}+i r\right) d r \\
& +\frac{1}{4} \hat{f}_{s}(0)\left(h_{\Gamma}-\Phi\left(\frac{d}{2}\right)\right) \\
& -\frac{h_{\Gamma}}{2 \pi} \int_{-\infty}^{\infty} \hat{f}_{s}(r) \frac{\Gamma^{\prime}}{\Gamma}(1+i r) d r \\
& +\kappa_{1} \int_{-\infty}^{\infty} \hat{f}_{s}(r) d r
\end{aligned}
$$

where $[Z(\Gamma)]$ is the order of the center $Z(\Gamma)$, the sum in the second line extends over all $\Gamma$-conjuigacy classes of elliptic and hyperbolic elements, $\gamma_{0}$ is a primitive element for $\gamma, h_{\Gamma}$ is the number of cusps, $\Phi$ is a scattering matrix and $\varphi$ is its determinant. $\kappa_{1}$ is the constant denoted in [2] as $\kappa_{5}$. The function $f_{s}(1)$ is defined by

$$
f_{s}(1)=\frac{1}{4 \pi} \int_{-\infty}^{\infty} \hat{f}_{s}(r) c(r)^{-1} c(-r)^{-1} d r
$$

with a c-function of Harish-Chandra $[3]$. Here it is expressed as

$$
c(r)^{-1}=\frac{\Gamma\left(\frac{d}{2}\right) \Gamma\left(i r+\frac{d}{2}\right)}{\Gamma(d) \Gamma(i r)} .
$$

We define the Selberg zeta function for $\Gamma$ as follows:

$$
Z(s):=\prod_{\left\{\gamma_{0}\right\}} \prod_{l \in L}\left\{1-\xi_{l}\left(h\left(\gamma_{0}\right)\right)^{-1} N\left(\gamma_{0}\right)^{-s}\right\}^{\kappa} \quad(\operatorname{Re}(s)>d),
$$

where the product on $\left\{\gamma_{0}\right\}$ is taken over all primitive hyperbolic conjugacy classes of $\Gamma, L$ is the semi-lattice of linear forms on $\mathfrak{a}$ of the form $\sum_{i=1}^{d} m_{i} \alpha_{i}$ with $\alpha_{1}, \cdots, \alpha_{d}$ being the elements of $P_{+}$and $m_{1}, \cdots, m_{d}$ being non-negative integers, and $\xi_{l}$ is the character of $\mathfrak{a}$ corresponding to $l, \xi_{l}(h)=\exp (l(\log h))$.

The logarithmic derivative of $Z(s)$ is as follows.

Proposition 2.2. The series

$$
\frac{Z^{\prime}}{Z}(s)=\kappa \sum_{\{\gamma\}} N(\gamma)^{\frac{d}{2}} \log N\left(\gamma_{0}\right) C(h(\gamma)) N(\gamma)^{-s}
$$

converges absolutely for each $s$ in $\operatorname{Re}(s)>d$, where $\{\gamma\}$ runs through the hyperbolic classes of $\Gamma, \gamma_{0}$ is a primitive element for $\gamma$, and $C(\cdot)$ is the positive function on $A_{\mathfrak{p}}$ defined by

$$
C(h(\gamma))=\varepsilon_{R}^{A}(h) \xi_{\rho}(h(\gamma))^{-1} \prod_{\alpha \in P_{+}}\left(1-\xi_{\alpha}(h(\gamma))^{-1}\right)^{-1}
$$


with

$$
\xi_{\rho}(h(\gamma))=\exp (\rho(\log h(\gamma)))=\exp \left(\frac{1}{2} \prod_{\alpha \in P_{+}} \alpha(\log (h(\gamma)))\right)
$$

and

$$
\varepsilon_{R}^{A}(h)=\operatorname{sign} \prod_{\alpha \in \mathfrak{a}}\left(1-\xi_{\alpha}(h)^{-1}\right) .
$$

We define

$$
\Lambda(\gamma):=\kappa N(\gamma)^{\frac{d}{2}} \log N\left(\gamma_{0}\right) C(h(\gamma))
$$

and

$$
\Psi(x):=\sum_{\{\gamma\} N(\gamma) \leq x} \Lambda(\gamma)
$$

where $\{\gamma\}$ runs through the hyperbolic classes of $\Gamma$.

\section{Propositions} 2.1 .

In this section, we will introduce the propositions as applications of Proposition

Let us begin with the existence problem for the eigenvalues of the Laplacian. We denote by $\mathbf{H}^{d+1}$ the $(d+1)$-dimensional hyperbolic space with the usual Riemannian hyperbolic metric of constant curvature -1 . The action of $G$ on the hyperbolic space $\mathbf{H}^{d+1}$ is transitive, so we have $\mathbf{H}^{d+1}=G / K$.

We denote the eigenvalues of the positive Laplacian $\Delta$ on $\mathbf{H}^{d+1}$ by $\lambda_{n}$ such that $0=\lambda_{0}<\lambda_{1} \leq \lambda_{2} \leq \cdots \leq \lambda_{M} \leq\left(\frac{d}{2}\right)^{2}<\lambda_{M+1} \cdots$, and let $t_{n}=\sqrt{\lambda_{n}-\left(\frac{d}{2}\right)^{2}}$.

Given a cofinite group $\Gamma \subset G$, we put

$$
N_{\Gamma}(T):=\sharp\left\{\lambda_{n} \mid \lambda_{n}<\left(\frac{d}{2}\right)^{2}+T^{2}\right\},
$$

the counting being done with multiplicities. The following result is obtained as a generalization of Weyl's law for a $(d+1)$-dimensional hyperbolic manifold.

Proposition 3.1. Let $\Gamma \subset S O_{e}(d+1,1)$ be a cofinite discrete subgroup, $\lambda_{n}$ be the eigenvalues of the Laplacian on $L^{2}\left(\Gamma \backslash \mathbf{H}^{d+1}\right)$ and $\varphi(s)$ be the scattering determinant. Then

$$
N_{\Gamma}(T)-\frac{1}{4 \pi} \int_{-T}^{T} \frac{\varphi^{\prime}}{\varphi}\left(\frac{d}{2}+i t\right) d t \sim C_{G} \operatorname{vol}(G / \Gamma) T^{d+1}
$$

as $T \rightarrow \infty$, where $C_{G}$ is the constant depending on $G$ such that

$$
C_{G} R^{-\frac{d+1}{2}}=\frac{[Z(\Gamma)]}{4 \pi \Gamma\left(\frac{d+1}{2}+1\right)}\left(\frac{\Gamma\left(\frac{d}{2}\right)}{\Gamma(d)}\right)^{2} \int_{-\infty}^{\infty} e^{-r^{2} R} \frac{\Gamma\left(i r+\frac{d}{2}\right) \Gamma\left(-i r+\frac{d}{2}\right)}{\Gamma(i r) \Gamma(-i r)} d r
$$

for any $R>0$.

Proof. Let $R>0$ and put $\hat{f}_{s}(r)=e^{-\left(\frac{d^{2}}{4}+r^{2}\right) R}$ in Proposition 2.1. From the discussion in [2, pp. 15-17], we have

$$
\begin{aligned}
\sum_{n=0}^{\infty} e^{-\left(\frac{d^{2}}{4}+r^{2}\right) R}-\frac{1}{4 \pi} \int_{-\infty}^{\infty} e^{-\left(\frac{d^{2}}{4}+r^{2}\right) R} \frac{\varphi^{\prime}}{\varphi}\left(\frac{d}{2}+i r\right) d r & \\
=C_{G} \Gamma\left(\frac{d+1}{2}+1\right) \operatorname{vol}(G / \Gamma) R^{-\frac{d+1}{2}}+o\left(R^{-\frac{d+1}{2}}\right) & \text { as } \quad R \rightarrow 0 .
\end{aligned}
$$


We write the left-hand side as a Laplace transform in the form $\int_{0}^{\infty} e^{-u R} d \alpha(u)$. Since the condition of a Tauberian theorem is satisfied [8, p. 51, Theorem 5], we have

$$
\alpha(u) \sim C_{G} \operatorname{vol}(G / \Gamma) u^{\frac{d+1}{2}} \quad \text { as } \quad u \rightarrow \infty .
$$

Taking into the account that

$$
\alpha(T)=N_{\Gamma}\left(T^{\frac{1}{2}}\right)-\frac{1}{4 \pi} \int_{-\sqrt{T}}^{\sqrt{T}} \frac{\varphi^{\prime}}{\varphi}\left(\frac{d}{2}+i t\right) d t+O(1)
$$

we obtain the proposition.

Here we introduce the Paley-Wiener theorem. For any complex $r$, we put

$$
H(r)=\int_{0}^{\infty} g^{\prime}(u) \exp (i r u) d u
$$

where $g$ is the function introduced in Section 2. Then, as in [2, we have the following lemma.

Lemma 3.2 ([2, Lemma 3.2]). The function $H$ is an entire function of $r$. Moreover, for each integer $n \geq 1$, we can find $c_{n}>0$ such that

$$
\begin{aligned}
& H(r) \leq c_{n}|r|^{-n} \quad \text { if } \operatorname{Im}(r) \geq 0 \text {, } \\
& \leq c_{n}|r|^{-n} \exp \left(\varepsilon_{0}|\operatorname{Im}(r)|\right) \quad \text { if } \operatorname{Im}(r)<0 .
\end{aligned}
$$

As an application of Proposition 2.1, combined with Lemma 3.2, we have the following property.

Proposition 3.3. Assume (1.4). Then we have for $\operatorname{Re}(s)>d$,

$$
\begin{aligned}
\frac{Z^{\prime}}{Z}(s)= & \frac{H\left(i\left(s-\frac{d}{2}\right)\right)}{s-\frac{d}{2}}+\sum_{\left|s-s_{n}\right|<1} \frac{H\left(i\left(s-s_{n}\right)\right)}{s-s_{n}} \\
& +\sum_{\left|s-\tilde{s}_{n}\right|<1} \frac{H\left(i\left(s-\tilde{s}_{n}\right)\right)}{s-\tilde{s}_{n}}+O\left(|s|^{d}+1\right),
\end{aligned}
$$

where $s_{n}=\frac{d}{2}+i t_{n}$ and $\tilde{s}_{n}=\frac{d}{2}-i t_{n}$ are the zeros of $Z(s)$ on $\operatorname{Re}(s)=\frac{d}{2}$.

Proof. In Proposition 2.1, we take the test function

$$
\hat{f}_{s}(r)=\frac{H\left(i s-i \frac{d}{2}+r\right)}{s-\frac{d}{2}-i r}+\frac{H\left(i s-i \frac{d}{2}-r\right)}{s-\frac{d}{2}+i r} .
$$


From Proposition 2.2, we have

$$
\begin{aligned}
\frac{Z^{\prime}}{Z}(s)= & \sum_{n=0}^{\infty}\left\{\frac{H\left(i s-i \frac{d}{2}+r\right)}{s-\frac{d}{2}-i r}+\frac{H\left(i s-i \frac{d}{2}-r_{n}\right)}{s-\frac{d}{2}+i r_{n}}\right\} \\
& -[Z(\Gamma)] \operatorname{vol}(G / \Gamma) f_{s}(1) \\
& -\frac{1}{4 \pi} \int_{-\infty}^{\infty}\left\{\frac{H\left(i s-i \frac{d}{2}+r\right)}{s-\frac{d}{2}-i r}+\frac{H\left(i s-i \frac{d}{2}-r\right)}{s-\frac{d}{2}+i r}\right\} \frac{\varphi^{\prime}}{\varphi}\left(\frac{d}{2}+i r\right) d r \\
& -\frac{1}{2} \frac{H\left(i s-i \frac{d}{2}\right)}{s-\frac{d}{2}}\left(h_{\Gamma}-\Phi\left(\frac{d}{2}\right)\right) \\
& +\frac{h_{\Gamma}}{2 \pi} \int_{-\infty}^{\infty}\left\{\frac{H\left(i s-i \frac{d}{2}+r\right)}{s-\frac{d}{2}-i r}+\frac{H\left(i s-i \frac{d}{2}-r\right)}{s-\frac{d}{2}+i r}\right\} \frac{\Gamma^{\prime}}{\Gamma}(1+i r) d r \\
& -\kappa_{1} \int_{-\infty}^{\infty} \frac{H\left(i s-i \frac{d}{2}+r\right)}{s-\frac{d}{2}-i r}+\frac{H\left(i s-i \frac{d}{2}-r\right)}{s-\frac{d}{2}+i r} d r,
\end{aligned}
$$

where

$$
\begin{aligned}
& f_{s}(1)=\frac{1}{4 \pi}\left(\frac{\Gamma\left(\frac{d}{2}\right)}{\Gamma(d)}\right)^{2} \int_{-\infty}^{\infty}\left\{\frac{H\left(i s-i \frac{d}{2}+r\right)}{s-\frac{d}{2}-i r}+\frac{H\left(i s-i \frac{d}{2}-r\right)}{s-\frac{d}{2}+i r}\right\} \\
& \frac{\Gamma\left(i r+\frac{d}{2}\right) \Gamma\left(-i r+\frac{d}{2}\right)}{\Gamma(i r) \Gamma(-i r)} d r .
\end{aligned}
$$

Changing the variables in $f_{s}(1)$, Lemma 3.2 and Stirling's formula for $\Gamma$ lead to

$$
f_{s}(1)=O\left(|s|^{d}+1\right) \text {. }
$$

From assumption (1.4), we have

$$
\frac{\varphi^{\prime}}{\varphi}\left(\frac{d}{2}+i r\right)=o\left(r^{d}\right)
$$

By (3.1), $\frac{\Gamma^{\prime}}{\Gamma}(1+i r)=O(\log r)$ and Lemma 3.2, other terms are also $O\left(|s|^{d}+1\right)$. We now have

$$
\frac{Z^{\prime}}{Z}(s)=\sum_{n=0}^{\infty}\left\{\frac{H\left(i s-i \frac{d}{2}+r_{n}\right)}{s-\frac{d}{2}-i r_{n}}+\frac{H\left(i s-i \frac{d}{2}-r_{n}\right)}{s-\frac{d}{2}+i r_{n}}\right\}+O\left(|s|^{d}+1\right) .
$$

Put $s_{n}=\frac{d}{2}+i r_{n}$ and $\tilde{s_{n}}=\frac{d}{2}-i r_{n}$. We obtain the proposition.

Proposition 3.3 leads to the following properties.

Proposition 3.4. Suppose (1.4); then we get

$$
\begin{aligned}
& \frac{Z^{\prime}}{Z}\left(\frac{d}{2}+\varepsilon+i t\right) \ll \frac{|t|^{d}}{\varepsilon} \quad(|t| \geq 2), \\
& \frac{Z^{\prime}}{Z}(d+\varepsilon) \ll \frac{1}{\varepsilon}, \\
& \frac{Z^{\prime}}{Z}(-\varepsilon+i t) \ll|t|^{d}+1, \\
& \left|\frac{Z^{\prime}}{Z}(s)\right| \ll|t|^{2 \max (0, d-\sigma)} \log |t| \quad\left(s=\sigma+i t, \sigma>1+\frac{1}{\log |t|},|t| \geq 2\right) .
\end{aligned}
$$


Moreover, for any $T$ there exists $\tau$ in $[T, T+1]$ such that

$$
\int_{0}^{d}\left|\frac{Z^{\prime}}{Z}(\sigma+i \tau)\right| d \sigma \ll T^{d} \log T .
$$

Proof. The equations (3.2) and (3.4) are obtained by a direct calculation with Proposition 3.3 and Lemma 3.2. We have the equation (3.3) by considering the range of convergence for $Z(s)$. We deduce (3.5) from (3.2) and (3.3) together with the Phragmen-Lindelöf principle. Thus, it remains only to show the inequality (3.6). We integrate the left-hand side of this relation over $\tau$ in

$$
\mathcal{T}:=\left\{\tau|T<\tau<T+1,| \tau-t_{n} \mid \geq T^{-(d+1)}\right\} .
$$

From the assumption (1.4) and Proposition 3.1, we have

$$
N_{\Gamma}(T) \sim C_{G} \operatorname{vol}(G / \Gamma) T^{d+1} \quad \text { as } T \rightarrow \infty .
$$

Taking this estimate (3.8), Proposition 3.3 and (3.7) into consideration, we have

$$
\int_{\mathcal{T}} \int_{0}^{d}\left|\frac{Z^{\prime}}{Z}(\sigma+i \tau)\right| d \sigma d \tau \ll T^{d} \log T .
$$

Since $|\mathcal{T}|=1+O\left(T^{-d}\right)$ for sufficiently large $T$, we have (3.6) for that $T$. For small $T$ the estimate is trivial.

We next introduce the functional equation for $Z(s)$. As a consequence from [2, Theorem 4.4], we have

Proposition 3.5. The following functional equation holds:

$$
\begin{aligned}
Z(d-s)= & Z(s)\left(\frac{\Gamma\left(1+\frac{d}{2}-s\right)}{\Gamma\left(1-\frac{d}{2}+s\right)}\right)^{\kappa h_{\Gamma}}[\varphi(d-s)]^{\kappa} \\
& \times \prod_{k=1}^{l}\left(\frac{s-\frac{d}{2}-q_{k}}{\frac{d}{2}-s-q_{k}}\right)^{\kappa b_{k}} \exp \left\{\int_{0}^{s-\frac{d}{2}} \Phi_{1}(t) d t+\kappa_{1}\left(s-\frac{d}{2}\right)\right\},
\end{aligned}
$$

where $\kappa$ is some positive integer mentioned in Section 2,

$$
\Phi_{1}(t):=\kappa \operatorname{vol}(G / \Gamma)[Z(\Gamma)]\left(\frac{\Gamma\left(\frac{d}{2}\right)}{\Gamma(d)}\right)^{2} \frac{\Gamma\left(\frac{d}{2}-t\right) \Gamma\left(\frac{d}{2}+t\right)}{\Gamma(t) \Gamma(-t)},
$$

$\kappa_{1}, h_{\Gamma}, \varphi$ are the same as in Proposition 2.1, $\left\{\frac{d}{2}+q_{k} \mid 1 \leq k \leq l\right\}$ are the finitely many poles of $\varphi$ in the interval $\left(0, \frac{d}{2}\right]$ and $b_{k}$ is the order of the pole at $q_{k}$.

Taking the logarithmic derivative of (3.9) gives

$$
\begin{aligned}
& -\frac{Z^{\prime}}{Z}(d-s)=\frac{Z^{\prime}}{Z}(s)+\kappa h_{\Gamma}\left\{-\frac{\Gamma^{\prime}}{\Gamma}\left(1+\frac{d}{2}-s\right)-\frac{\Gamma^{\prime}}{\Gamma}\left(1-\frac{d}{2}+s\right)\right\} \\
& -\kappa \frac{\varphi^{\prime}}{\varphi}(d-s)+\sum_{k=1}^{l} \kappa b_{k}\left(\frac{1}{s-\frac{d}{2}-q_{k}}+\frac{1}{\frac{d}{2}-s-q_{k}}\right)+\Phi_{1}\left(s-\frac{d}{2}\right)+\kappa_{1} .
\end{aligned}
$$

From Stirling's formula, we have

$$
\Phi_{1}\left(s-\frac{d}{2}\right)=O\left(|s|^{d}\right) .
$$


By this estimate and

$$
\begin{aligned}
& \frac{\Gamma^{\prime}}{\Gamma}\left(1+\frac{d}{2}-s\right)+\frac{\Gamma^{\prime}}{\Gamma}\left(1-\frac{d}{2}+s\right)=O(\log s) \\
& \frac{\varphi^{\prime}}{\varphi}(d-s)=o\left(|s|^{d}\right)
\end{aligned}
$$

we have

$$
\frac{Z^{\prime}}{Z}(s)+\frac{Z^{\prime}}{Z}(d-s)=O\left(|s|^{d}\right)
$$

\section{ProOF}

We are now ready to prove the main theorem, Theorem 1.2, Let us begin by describing the explicit formula for $\Psi(x)$. We define $\Psi_{d}(x)$ for $d \in \mathbf{N}$ recursively as $\Psi_{d}(x):=\int_{1}^{x} \Psi_{d-1}(t) d t$, where $\Psi_{0}(x)=\Psi(x)$.

Theorem 4.1 ([5, Theorem A]). Let $a_{1}, a_{2}, \cdots$ be a real sequence which increases (in the wide sense) and has the limit infinity, and let

$$
C(x)=\sum_{a_{n} \leq x} c_{n}
$$

where the $c_{n}$ may be real or complex, and the notation indicates a summation over the (finite) set of positive integers $n$ for which $a_{n} \leq x$. Then, if $X \geq a_{1}$ and $\phi(x)$ has a continuous derivative, we have

$$
\sum_{a_{n} \leq X} c_{n} \phi\left(a_{n}\right)=-\int_{a_{1}}^{X} C(x) \phi^{\prime}(x) d x+C(X) \phi(X) .
$$

If, further, $C(X) \phi(X) \rightarrow 0$ as $X \rightarrow \infty$, then

$$
\sum_{n=1}^{\infty} c_{n} \phi\left(a_{n}\right)=-\int_{a_{1}}^{\infty} C(x) \phi^{\prime}(x) d x
$$

provided that either side is convergent.

By this theorem, the following equation is obtained:

$$
d ! \Psi_{d}(x)=\sum_{N(\gamma) \leq x} \Lambda(\gamma)(x-N(\gamma))^{d} .
$$

The cases of $d=1$ and $d=2$ are in [7].

Theorem 4.2 ([5, p. 31, Theorem B]). If $k$ is a positive integer, $c>0, y>0$, then

$$
\frac{1}{2 \pi i} \int_{c-\infty s i}^{c+\infty i} \frac{y^{s} d s}{s(s+1) \cdots(s+k)}= \begin{cases}0 & (y \leq 1), \\ \frac{1}{k !}\left(1-\frac{1}{y}\right)^{k} & (y>1) .\end{cases}
$$

This theorem gives

$$
\Psi_{d}(x)=\frac{1}{2 \pi i} \int_{c-\infty i}^{c+\infty i} \frac{x^{s+d}}{s(s+1) \cdots(s+d)} \frac{Z^{\prime}}{Z}(s) d s
$$

for $c>2$. We choose $c=\frac{3}{2} d(d \geq 2)$; then the following theorem holds. 
Theorem 4.3. We have

$$
\begin{aligned}
\Psi_{d}(x) & =\sum_{n=0}^{M} \frac{x^{s_{n}+d}}{s_{n}\left(s_{n}+1\right) \cdots\left(s_{n}+d\right)}+\sum_{n=0}^{M} \frac{x^{\tilde{s}_{n}+d}}{\tilde{s}_{n}\left(\tilde{s}_{n}+1\right) \cdots\left(\tilde{s}_{n}+d\right)} \\
& +\sum_{t_{n} \geq 0} \frac{x^{s_{n}+d}}{s_{n}\left(s_{n}+1\right) \cdots\left(s_{n}+d\right)}+\sum_{t_{n}>0} \frac{x^{\tilde{s}_{n}+d}}{\tilde{s}_{n}\left(\tilde{s}_{n}+1\right) \cdots\left(\tilde{s}_{n}+d\right)}+O\left(x^{\frac{3}{2} d}\right),
\end{aligned}
$$

where $s_{n}=\frac{d}{2}+i t_{n}, \tilde{s}_{n}=\frac{d}{2}-i t_{n}$ are the zeros of $Z(s)$.

Proof. We suppose $T$ is large. Let $A:=N+\frac{1}{2}$, where $N$ is a positive integer. By applying Cauchy's theorem with

$$
R(A, T):=\left\{z \in \mathbf{C} \mid-A<\operatorname{Re}(z) \leq \frac{3}{2} d,-T \leq \operatorname{Im}(z) \leq T\right\},
$$

we have

$$
\begin{aligned}
& \frac{1}{2 \pi i} \int_{\frac{3}{2} d-i T}^{\frac{3}{2} d+i T} \frac{x^{s+d}}{s(s+1) \cdots(s+d)} \frac{Z^{\prime}}{Z}(s) \\
& =\frac{1}{2 \pi i}\left(\int_{-A-i T}^{-A+i T}+\int_{-A+i T}^{\frac{3}{2} d+i T}-\int_{-A-i T}^{\frac{3}{2} d-i T}\right) \frac{x^{s+d}}{s(s+1) \cdots(s+d)} \frac{Z^{\prime}}{Z}(s) d s \\
& \quad+\sum_{z \in R(A, T)} \operatorname{Res}_{s=z}\left(\frac{x^{s+d}}{s(s+1) \cdots(s+d)} \frac{Z^{\prime}}{Z}(s)\right) .
\end{aligned}
$$

For the integral parts of the right-hand side, by using the functional equation (3.10) we have

$$
\frac{1}{2 \pi i} \int_{-A-i T}^{-A+i T} \frac{x^{s+d}}{s(s+1) \cdots(s+d)} \frac{Z^{\prime}}{Z}(s) d s=O\left(x^{d-A}\right)
$$

and

$$
\begin{aligned}
\frac{1}{2 \pi i}\left(\int_{-A+i T}^{\frac{3}{2} d+i T}-\right. & \left.\int_{-A-i T}^{\frac{3}{2} d-i T}\right) \frac{x^{s+d}}{s(s+1) \cdots(s+d)} \frac{Z^{\prime}}{Z}(s) d s \\
& =O\left(\frac{x^{\frac{3}{2} d}-x^{d-A}}{T \log x}\right)+O\left(\int_{\frac{d}{2}+i T}^{\frac{3}{2} d+i T} \frac{x^{\sigma+d}}{T^{d+1}}\left|\frac{Z^{\prime}}{Z}(s)\right||d s|\right),
\end{aligned}
$$

where $\sigma=\operatorname{Re}(s)$ in the last term.

On the other hand, equation (4.1) for $c=\frac{3}{2} d$ shows

$$
\Psi_{d}(x)=\frac{1}{2 \pi i} \int_{\frac{3}{2} d-i T}^{\frac{3}{2} d+i T} \frac{x^{s+d}}{s(s+1) \cdots(s+d)} \frac{Z^{\prime}}{Z}(s) d s+O\left(\frac{x^{\frac{5}{2} d}}{T^{d}}\right) .
$$

Combining (4.2), (4.3), (4.4), (4.5), we have

$$
\begin{aligned}
\Psi_{d}(x)+O\left(\frac{x^{\frac{5}{2} d}}{T^{d}}\right)=O\left(x^{d-A}\right) & +O\left(\frac{x^{\frac{3}{2} d}}{T \log x}\right)+O\left(\int_{\frac{d}{2}+i T}^{\frac{3}{2} d+i T} \frac{x^{\sigma+d}}{T^{d+1}}\left|\frac{Z^{\prime}}{Z}(s)\right||d s|\right) \\
& +\sum_{z \in R(A, T)} \operatorname{Res}_{s=z}\left(\frac{x^{s+d}}{s(s+1) \cdots(s+d)} \frac{Z^{\prime}}{Z}(s)\right) .
\end{aligned}
$$


We now estimate the integral term on the right-hand side of (4.6). Since

$$
\int_{\frac{d}{2}+i T}^{\frac{3}{2} d+i T} \frac{x^{\sigma+d}}{T^{d+1}}\left|\frac{Z^{\prime}}{Z}(s)\right||d s|=\int_{\frac{d}{2}}^{\frac{3}{2} d} \frac{x^{\sigma+d}}{T^{d+1}}\left|\frac{Z^{\prime}}{Z}(\sigma+i T)\right| d \sigma,
$$

by Proposition 3.4 it is estimated by

$$
\int_{\frac{d}{2}}^{\frac{d}{2}+\varepsilon} \frac{x^{\sigma+d}}{T^{d+1}} T^{d} d \sigma+\int_{\frac{d}{2}+\varepsilon}^{d} \frac{x^{\sigma+d}}{T^{d+1}} T^{2(d-\sigma)} d \sigma+\int_{d}^{\frac{3}{2} d} \frac{x^{\sigma+d}}{T^{d+1}} \frac{1}{\varepsilon} d \sigma .
$$

After computing these integrals, we have

$$
\int_{\frac{d}{2}+i T}^{\frac{3}{2} d+i T} \frac{x^{\sigma+d}}{T^{d+1}}\left|\frac{Z^{\prime}}{Z}(s)\right||d s| \ll \frac{x^{\frac{5}{2} d}}{T(\log x-2 \log T)} .
$$

This estimate, calculations of residues at $s=0,-1, \cdots,-d, s_{n}, \tilde{s}_{n}, \rho_{n}, \tilde{\rho}_{n}$ and (4.6) give

$$
\begin{aligned}
\Psi_{d}(x)+O\left(\frac{x^{\frac{5}{2} d}}{T^{d}}\right) & =O\left(x^{d-A}\right)+O\left(\frac{x^{\frac{3}{2} d}}{T \log x}\right)+O\left(\frac{x^{\frac{5}{2} d}}{T(\log x-2 \log T)}\right) \\
& +\sum_{n=0}^{M} \frac{x^{s_{n}+d}}{s_{n}\left(s_{n}+1\right) \cdots\left(s_{n}+d\right)}+\sum_{n=0}^{M} \frac{x^{\tilde{s}_{n}+d}}{\tilde{s}_{n}\left(\tilde{s}_{n}+1\right) \cdots\left(\tilde{s}_{n}+d\right)} \\
& +\sum_{t_{n} \geq 0} \frac{x^{s_{n}+d}}{s_{n}\left(s_{n}+1\right) \cdots\left(s_{n}+d\right)}+\sum_{t_{n}>0} \frac{x^{\tilde{s}_{n}+d}}{\tilde{s}_{n}\left(\tilde{s}_{n}+1\right) \cdots\left(\tilde{s}_{n}+d\right)} \\
& +O\left(x^{\frac{3}{2} d}\right) .
\end{aligned}
$$

Letting both $A$ and $T$ go to $\infty$, we obtain the theorem.

Our next goal is to show an $\Omega$-result for

$$
P(x):=\Psi(x)-\left(\sum_{n=0}^{M} \frac{x^{s_{n}}}{s_{n}}+\sum_{n=0}^{M} \frac{x^{\tilde{s}_{n}}}{\tilde{s}_{n}}+R(x)\right),
$$

where $R(x)$ is the term from the residues at $s=0,-1, \cdots,-d, \rho_{n}, \tilde{\rho}_{n}$.

We define $P_{i}(x)$ for $i \in \mathbf{N}$ recursively as

$$
P_{i}(x):=\int_{0}^{x} P_{i-1}(t) d t .
$$

Here $P_{1}(x):=\int_{0}^{x} P(t) d t$. Further we define

$$
\mathcal{P}(x):=P(x)-N_{\Gamma}(0) x .
$$

Then we have the following.

Lemma 4.4. There exists $c_{1} \in \mathbf{C}$ such that

$$
c_{1}+\int_{1}^{v} \frac{\mathcal{P}\left(e^{u}\right)}{e^{\frac{d}{2} u}} d u=\sum_{t_{n}>0} \frac{e^{\left(s_{n}-\frac{d}{2}\right) v}}{s_{n}\left(s_{n}-\frac{d}{2}\right)}+\sum_{t_{n}>0} \frac{e^{\left(\tilde{s}_{n}-\frac{d}{2}\right) v}}{\tilde{s}_{n}\left(\tilde{s}_{n}-\frac{d}{2}\right)} .
$$

Proof. Put

$$
\mathcal{P}_{i}(x):=P_{i}(x)-\frac{1}{(i+1) !} N_{\Gamma}(0) x^{i+1} \quad(i=1, \cdots, d) .
$$


For

$$
F(v):=d_{1}+\int_{1}^{v} \frac{\mathcal{P}\left(e^{u}\right)}{e^{\frac{d}{2} u}} d u \text { for } v \geq 1,
$$

where $d_{1} \in \mathbf{C}$ is unspecified temporarily, changing of variables with $x=e^{u}$ gives us that

$$
F(v)=d_{1}+\int_{e}^{e^{v}} \frac{\mathcal{P}(x)}{x^{\frac{d}{2}+1}} d x .
$$

By integration by parts repeatedly, we have

$F(v)=d_{1}+f_{1}+\frac{\mathcal{P}\left(e^{v}\right)}{e^{\left(\frac{d}{2}+1\right) v}}+\sum_{i=2}^{d} \frac{\Gamma\left(\frac{3}{2} d+i\right)}{\Gamma\left(\frac{d}{2}+1\right)} \mathcal{P}_{i}\left(e^{v}\right) e^{-\left(\frac{d}{2}+i\right) v}+\frac{\Gamma\left(\frac{3}{2} d+1\right)}{\Gamma\left(\frac{d}{2}+1\right)} \int_{e}^{e^{v}} \frac{\mathcal{P}_{d}(x)}{x^{\frac{3}{2} d+1}} d x$,

for some constant $f_{1}$. Since Theorem 4.3 implies

$$
\mathcal{P}_{d}(x)=\sum_{t_{n} \geq 0} \frac{x^{s_{n}+d}}{s_{n}\left(s_{n}+1\right) \cdots\left(s_{n}+d\right)}+\sum_{t_{n}>0} \frac{x^{\tilde{s}_{n}+d}}{\tilde{s}_{n}\left(\tilde{s}_{n}+1\right) \cdots\left(\tilde{s}_{n}+d\right)},
$$

we have

$$
\begin{aligned}
\int_{e}^{e^{v}} \frac{\mathcal{P}_{d}(x)}{x^{\frac{3}{2} d+1}} d x= & \sum_{t_{n} \geq 0} \frac{x^{\left(s_{n}-\frac{d}{2}\right) v}}{s_{n}\left(s_{n}+1\right) \cdots\left(s_{n}+d\right)\left(s_{n}-\frac{d}{2}\right)} \\
& +\sum_{t_{n}>0} \frac{x^{\tilde{s}_{n}-\frac{d}{2}}}{\tilde{s}_{n}\left(\tilde{s}_{n}+1\right) \cdots\left(\tilde{s}_{n}+d\right)\left(\tilde{s}_{n}-\frac{d}{2}\right)}+f_{2}
\end{aligned}
$$

and

$$
\begin{aligned}
\frac{\mathcal{P}_{m}\left(e^{v}\right)}{e^{\left(\frac{d}{2}+m\right) v}}= & \sum_{t_{n} \geq 0} \frac{e^{\left(s_{n}-\frac{d}{2}\right) v}}{s_{n}\left(s_{n}+1\right) \cdots\left(s_{n}+m\right)} \\
& +\sum_{t_{n}>0} \frac{e^{\left(\tilde{s}_{n}-\frac{d}{2}\right) v}}{\tilde{s}_{n}\left(\tilde{s}_{n}+1\right) \cdots\left(\tilde{s}_{n}+m\right)} \quad(m=1,2, \cdots, d) .
\end{aligned}
$$

Inserting these calculations into (4.10) gives us

$$
F(v)=d_{1}+f_{3}+\sum_{t_{n}>0}\left(\frac{e^{\left(s_{n}-\frac{d}{2}\right) v}}{s_{n}\left(s_{n}-\frac{d}{2}\right)}+\frac{e^{\left(\tilde{s}_{n}-\frac{d}{2}\right) v}}{\tilde{s}_{n}\left(\tilde{s}_{n}-\frac{d}{2}\right)}\right) .
$$

By taking $d_{1}=-f_{3}=: c_{1}$, we have the lemma.

In what follows we put

$$
F(v):=c_{1}+\int_{1}^{v} \frac{\mathcal{P}\left(e^{u}\right)}{e^{\frac{d}{2} u}} d u .
$$

From Lemma 4.4 we easily find the following.

Lemma 4.5. There exists $c_{2} \in \mathbf{C}$ such that

$$
c_{2}+\int_{1}^{v} F(u) d u=\sum_{t_{n}>0} \frac{e^{\left(s_{n}-\frac{d}{2}\right) v}}{s_{n}\left(s_{n}-\frac{d}{2}\right)^{2}}+\sum_{t_{n}>0} \frac{e^{\left(\tilde{s}_{n}-\frac{d}{2}\right) v}}{\tilde{s}_{n}\left(\tilde{s}_{n}-\frac{d}{2}\right)^{2}} .
$$


In what follows we put

$$
F_{2}(v):=c_{2}+\int_{1}^{v} F(u) d u .
$$

In the same way, we define $F_{d}(v)$ by

$$
F_{d}(v):=c_{d}+\int_{1}^{v} F_{d-1}(u) d u
$$

for some constants $c_{d}(d \geq 2)$.

We now introduce the function $k(x):=\left(\frac{\sin \pi x}{\pi x}\right)^{2}$, which has the following properties.

Lemma 4.6. Let $k(x):=\left(\frac{\sin \pi x}{\pi x}\right)^{2}$. Then

a) $k(x)$ is a $C^{\infty}$-function on $\mathbf{R}$;

b) $k(x), k^{\prime}(x)$ and $k^{(n)}(x)$ for $(n=1,2, \cdots, d)$ are all $O\left(x^{-2}\right)$ when $|x| \rightarrow \infty$;

c) $\int_{-\infty}^{\infty} k(x) e^{i u x} d x=\max \left[0,1-\frac{|u|}{2 \pi}\right]$.

Proof. Every statement except for b) is proved by Hejhal [4, p. 264, Lemma 16.9]. In b), the cases of $n=1,2,3$ are obtained in [7, Lemma 3.14]. For other cases, the relevant property is also deduced by the same method.

Lemma 4.7. Let $r, A$ and $Y$ be positive constants. We have

$$
\begin{aligned}
\int_{1}^{r+A} \frac{\mathcal{P}\left(e^{v}\right)}{e^{\frac{d}{2} v}} k(Y(v-r)) d v= & -\frac{2}{Y} \sum_{0<t_{n} \leq 2 \pi Y} \frac{\sin \left(t_{n} r\right)}{t_{n}}\left(1-\frac{t_{n}}{2 \pi Y}\right)+O\left(\frac{Y^{d-2}}{r^{2}}\right) \\
& +O\left(\frac{Y^{d-1}}{r^{3}}\right)+O\left(\frac{Y^{d-1}}{A^{3}}\right)+O\left(Y^{d+1}\right) .
\end{aligned}
$$

This lemma is generalization of [7, Lemma 3.15]. The method of the proof is similar.

Proof. For convenience, we assume $A$ and $Y$ are integers. Using integration by parts repeatedly, we have

$$
\begin{aligned}
\int_{1}^{r+A} \frac{\mathcal{P}\left(e^{v}\right)}{e^{\frac{d}{2} v}} & k(Y(v-r)) d v \\
= & O\left(\frac{Y^{d-2}}{r^{2}}\right)+(-Y)^{d+1} \int_{1}^{r+A} F_{d+1}(v) k^{(d+1)}(Y(v-r)) d v .
\end{aligned}
$$

The function $F_{d+1}$ has been defined for all $v \in \mathbf{R}$. Since $F_{d+1}(v)$ is uniformly bounded, we have

$$
Y^{d+1} \int_{r+A}^{\infty}\left|F_{d+1}(v) k^{(d+1)}(Y(v-r))\right| d v=O\left(Y^{d-1}\left(\frac{1}{r^{3}}+\frac{1}{A^{3}}\right)\right)
$$

and

$$
Y^{d+1} \int_{-\infty}^{1}\left|F_{d+1}(v) k^{(d+1)}(Y(v-r))\right| d v=O\left(\frac{Y^{d-1}}{r^{3}}\right) .
$$


Therefore (4.12) becomes

$$
\begin{aligned}
\int_{1}^{r+A} \frac{\mathcal{P}\left(e^{v}\right)}{e^{\frac{d}{2} v}} k(Y(v-r)) d v & =O\left(\frac{Y^{d-2}}{r^{2}}\right)+O\left(\frac{Y^{d-1}}{r^{3}}\right)+O\left(\frac{Y^{d-1}}{A^{3}}\right) \\
& +(-Y)^{d+1} \int_{-\infty}^{\infty} F_{d+1}(v) k^{(d+1)}(Y(v-r)) d v .
\end{aligned}
$$

From Lemma 4.5 and the definition of $F_{d}$, we have

$$
F_{d+1}(v)=\sum_{t_{n}>0} \frac{e^{\left(s_{n}-\frac{d}{2}\right) v}}{s_{n}\left(s_{n}-\frac{d}{2}\right)^{d+1}}+\sum_{t_{n}>0} \frac{e^{\left(\tilde{s}_{n}-\frac{d}{2}\right) v}}{\tilde{s}_{n}\left(\tilde{s}_{n}-\frac{d}{2}\right)^{d+1}}
$$

where these sums converge uniformly on $\mathbf{R}$. Hence, in the last term of (4.13), we can change the order of integration and summation. After integrating term by term, it becomes

$$
-\sum_{t_{n}>0} \int_{-\infty}^{\infty}\left(\frac{e^{\left(s_{n}-\frac{d}{2}\right) v}}{s_{n}}+\frac{e^{\left(\tilde{s}_{n}-\frac{d}{2}\right) v}}{\tilde{s}_{n}}\right) k(Y(v-r)) d v .
$$

By changing variables with $X:=Y(v-r)$ and using Lemma 4.6, this term is expressed as

$$
-\frac{2}{Y} \sum_{0<t_{n} \leq 2 \pi Y} \frac{\frac{d}{2} \cos \left(t_{n} r\right)+t_{n} \sin \left(t_{n} r\right)}{\left(\frac{d}{2}\right)^{2}+t_{n}{ }^{2}}\left(1-\frac{t_{n}}{2 \pi Y}\right) .
$$

Under the assumption (1.4), from (3.8) we have

$$
\sum_{0<t_{n} \leq R} \frac{1}{\left|s_{n}\right|^{2}}=O\left(R^{d-1}\right)
$$

and we notice

$$
\frac{t_{n}}{\left(\frac{d}{2}\right)^{2}+t_{n}^{2}}<\frac{1}{t_{n}}-\frac{1}{t_{n}\left(t_{n}^{2}+1\right)} .
$$

Taking them into the consideration, the lemma follows.

We now consider $t_{n}$ in the range of $(0,2 \pi Y]$.

Lemma 4.8. For $Y$ large, there exist some constants $k_{1}$ and $k_{2}$ which satisfy

$$
e^{k_{1} Y^{d+1}} \leq \prod_{0<t_{n} \leq 2 \pi Y}\left(1+\frac{100 \pi Y}{t_{n}}\right) \leq e^{k_{2} Y^{d+1}} .
$$

The proof is deduced from the same method in [7, Lemma 3.16].

Lemma 4.9 ([4, p. 266 Lemma 16.10]). Let $a_{1}, \cdots, a_{n}$ be real numbers. Suppose that $T_{0}, \delta_{1}, \cdots, \delta_{n}$ are positive numbers. Then there exists integers $x_{1}, \cdots, x_{n}$ and a number $r$ such that:

$$
\begin{gathered}
\left|t a_{k}-x_{k}\right| \leq \delta_{k} \quad \text { for } \quad 1 \leq k \leq n, \\
T_{0} \leq r \leq T_{0} \prod_{k=1}^{n}\left(1+\frac{1}{\delta_{k}}\right) .
\end{gathered}
$$

Applying the above property for real number sequence, we have the following lemma by the same way as [7, Lemma 3.18]. 
Lemma 4.10. There exists $r_{0}$ such that

$$
r_{0} t_{n}=2 \pi I+\varepsilon_{n} \quad \text { for } \quad 0<t_{n} \leq 2 \pi Y,
$$

where $I$ is an integer and $\left|\varepsilon_{n}\right| \leq \frac{t_{n}}{50} Y$, and

$$
e^{k_{3} Y^{d+1}} \leq r_{0} \leq e^{2 k_{3} Y^{d+1}}
$$

for some positive constant $k_{3}>0$.

Combining above results, we can obtain the following theorem about the $\Omega$-result for $P(x)$.

Theorem 4.11. Assume (1.4). Then we have

$$
P(x)=\Omega_{ \pm}\left(x^{\frac{d}{2}}(\log \log x)^{\frac{1}{d+1}}\right) .
$$

Proof. Put

$$
r_{1}=r_{0}-\frac{1}{4 \pi Y}
$$

For $-\frac{t_{n}}{2 \pi Y} \leq \varepsilon_{n}-\frac{t_{n}}{4 \pi Y} \leq\left(\frac{1}{50 Y}-\frac{t_{n}}{4 \pi Y}\right) t_{n}$, since Lemma 4.10 implies that

$$
r_{1} t_{n}=2 \pi I+\varepsilon_{n}-\frac{t_{n}}{4 \pi Y}
$$

we have

$$
\sin \left(r_{1} t_{n}\right)=\sin \left(\varepsilon_{n}-\frac{t_{n}}{4 \pi Y}\right) .
$$

When we assume (1.4), we find enough $t_{n}$, so that from (4.17) there exists $k_{4}>0$ which satisfies

$$
-\sin \left(r_{1} t_{n}\right) \geq k_{4} \frac{t_{n}}{Y}
$$

for large $Y$. Hence, we find $k_{5}>0$ such that

$$
-\frac{2}{Y} \sum_{0<t_{n} \leq 2 \pi Y} \frac{\sin \left(r_{1} t_{n}\right)}{t_{n}}\left(1-\frac{t_{n}}{2 \pi Y}\right) \geq k_{5} .
$$

Considering Lemma 4.7, there exists some constant $k_{6}>0$ which satisfies

$$
\int_{1}^{r_{1}+A} \frac{\mathcal{P}\left(e^{v}\right)}{e^{\frac{d}{2} v}} k\left(Y\left(v-r_{1}\right)\right) d v \geq k_{6},
$$

where $A$ and $Y$ are kept sufficiently large and $A$ is independent of $Y$. Letting

$$
X:=\sup \left\{\frac{\mathcal{P}\left(e^{v}\right)}{e^{\frac{d}{2} v}} \mid 1 \leq v \leq A+r_{1}\right\},
$$

we deduce that

$$
X \int_{-\infty}^{\infty} k\left(Y\left(v-r_{1}\right)\right) d v \geq X \int_{1}^{r_{1}+A} k(Y(v-r)) d v \geq k_{6} .
$$

From $\int_{-\infty}^{\infty} k\left(Y\left(v-r_{1}\right)\right) d v=O\left(\frac{1}{Y}\right)$, this gives us

$$
X \geq k_{6}{ }^{\prime} Y \quad\left(k_{6}{ }^{\prime}>0\right) .
$$

From (4.16) we find $k_{7}$ and $k_{8}$ such that $k_{7} Y \leq\left(\log r_{1}\right)^{\frac{1}{d+1}} \leq k_{8} Y$, and it shows there exists $k_{9}>0$ which satisfies

$$
X \geq k_{9}\left(\log r_{1}\right)^{\frac{1}{d+1}} .
$$


Therefore, we have

$$
\varlimsup_{v \rightarrow \infty} \frac{\mathcal{P}\left(e^{v}\right)}{e^{\frac{d}{2} v}(\log v)^{\frac{1}{d+1}}} \geq k_{9} .
$$

Putting $x=e^{v}$, we have $\mathcal{P}(x)=\Omega_{+}\left(x^{\frac{d}{2}}(\log \log x)^{\frac{1}{d+1}}\right)$. The $\Omega_{-}$-result is proved similarly by using $r_{2}=r_{0}+\frac{1}{4 \pi Y}$. Then we have

$$
\mathcal{P}(x)=\Omega_{ \pm}\left(x^{\frac{d}{2}}(\log \log x)^{\frac{1}{d+1}}\right) .
$$

From the definition of $\mathcal{P}(x)$, this concludes the proof of the theorem.

Next, we will clarify the relation between $\pi_{\Gamma}(x)$ and $P(x)$.

From Proposition 2.2, we note that

$$
\sum_{\{\gamma\}} \log N\left(\gamma_{0}\right)-C(h(\gamma)) N(\gamma)^{-\left(s+\frac{d}{2}\right)} \sim \frac{1}{s-\frac{d}{2}} \quad \text { as } \quad s \rightarrow \frac{d}{2},
$$

where $\{\gamma\}$ runs through the hyperbolic classes of $\Gamma$ and $\gamma_{0}$ is the primitive element of $\gamma$. Hence, we have

$$
\sum\{\gamma\} N(\gamma) \leq x \log N\left(\gamma_{0}\right) C(h(\gamma))=O(\log x),
$$

which implies

$$
\sum\{\gamma\} N(\gamma) \leq x \frac{\log N\left(\gamma_{0}\right)}{N(\gamma)^{\frac{d}{2}}}=O(\log x),
$$

by the definition of $C(h(\gamma))$. This estimate gives the following.

Lemma 4.12. We have

$$
\sum_{N\left(\gamma_{0}\right) \leq x} \log N\left(\gamma_{0}\right)-\Psi(x)=O\left(x^{\frac{d}{2}} \log x\right) .
$$

Proof. It suffices to prove that

$$
\sum_{N\left(\gamma_{0}\right) \leq x} \frac{\log N\left(\gamma_{0}\right)}{N\left(\gamma_{0}\right)^{\frac{d}{2}}}-\sum_{N(\gamma) \leq x} \log N\left(\gamma_{0}\right) C(h(\gamma))=O(\log x) \quad \text { as } \quad x \rightarrow \infty
$$

From the definition of $C(h(\gamma))$, we may find constants $c_{i}$ such that

$$
\sum_{N\left(\gamma_{0}\right) \leq x} \log N\left(\gamma_{0}\right) C\left(h\left(\gamma_{0}^{i}\right)\right) \leq c_{i} \sum_{N\left(\gamma_{0}\right) \leq x} \frac{\log N\left(\gamma_{0}\right)}{N\left(\gamma_{0}\right)^{\frac{i}{2} d}} .
$$

Splitting off the contribution of the primitive elements to the second term in (4.20), using (4.21) we find a constant $C>0$ such that

$$
\begin{aligned}
& \sum_{N(\gamma) \leq x} \log N\left(\gamma_{0}\right) C(h(\gamma))-\sum_{N\left(\gamma_{0}\right) \leq x} \log N\left(\gamma_{0}\right) C\left(h\left(\gamma_{0}\right)\right) \mid \\
& \leq C\left\{\sum_{N\left(\gamma_{0}\right) \leq x} \frac{\log N\left(\gamma_{0}\right)}{N\left(\gamma_{0}\right)^{d}}+\sum_{N\left(\gamma_{0}\right)^{2} \leq x} \frac{\log N\left(\gamma_{0}\right)}{N\left(\gamma_{0}\right)^{\frac{3}{2} d}}+\cdots+\sum_{N\left(\gamma_{0}\right)^{k} \leq x} \frac{\log N\left(\gamma_{0}\right)}{N\left(\gamma_{0}\right)^{\frac{k}{2} d}}\right\} .
\end{aligned}
$$

The right-hand side of (4.22) has at most $O(\log x)$ terms in total. Since we have

$$
\sum_{N\left(\gamma_{0}\right) \leq x} \frac{\log N\left(\gamma_{0}\right)}{N\left(\gamma_{0}\right)^{\frac{i}{2} d}} \leq \sum_{\left\{\gamma_{0}\right\}} \frac{\log N\left(\gamma_{0}\right)}{N\left(\gamma_{0}\right)^{\frac{3}{2} d}}<\infty \quad \text { for } \quad i \geq 3
$$


the estimate (4.19) leads to

$$
\left|\sum_{N(\gamma) \leq x} \log N(\gamma) C(h(\gamma))-\sum_{N\left(\gamma_{0}\right) \leq x} \log N\left(\gamma_{0}\right) C\left(h\left(\gamma_{0}\right)\right)\right|=O(\log x) .
$$

On the other hand, there exists a constant $K>0$ which satisfies

$$
\begin{aligned}
\left.|| C\left(h\left(\gamma_{0}\right)\right)\right|^{2}-\left|N\left(\gamma_{0}\right)^{-\frac{d}{2}}\right|^{2} \mid & =N\left(\gamma_{0}\right)^{-d}\left|\prod_{\alpha}\left(1-\xi_{\alpha}(h(\gamma))\right)^{-2}\right| \\
& \leq K N\left(\gamma_{0}\right)^{-d} \quad(d \geq 2),
\end{aligned}
$$

which gives

$$
\left|\sum_{N\left(\gamma_{0}\right) \leq x} \log N\left(\gamma_{0}\right) C\left(h\left(\gamma_{0}\right)\right)-\sum_{N\left(\gamma_{0}\right) \leq x} \frac{\log N\left(\gamma_{0}\right)}{N\left(\gamma_{0}\right)^{\frac{d}{2}}}\right|=O(\log x) .
$$

From (4.23) and (4.24), we have the desired estimate (4.20).

Let

$$
P_{0}(x):=\sum_{N\left(\gamma_{0}\right) \leq x} \log N\left(\gamma_{0}\right)-\left(\sum_{n=0}^{M} \frac{x^{s_{n}}}{s_{n}}+\sum_{n=0}^{M} \frac{x^{\tilde{s}_{n}}}{\tilde{s}_{n}}\right)
$$

and

$$
Q(x)=\sum_{N\left(\gamma_{0}\right) \leq x} \log N\left(\gamma_{0}\right)-\Psi(x),
$$

where $\gamma_{0}$ is the primitive element in $\Gamma$. Then from (4.7), we have

$$
P_{0}(x)=P(x)+Q(x)+R(x) .
$$

From the definition of $\pi_{\Gamma}(x)$, we have

$$
\int_{2}^{x} \frac{d P_{0}(t)}{\log t}=\pi_{\Gamma}(x)-\left(\sum_{n=0}^{M} \operatorname{li}\left(x^{s_{n}}\right)+\sum_{n=0}^{M} \operatorname{li}\left(x^{\tilde{s}_{n}}\right)\right) .
$$

From Lemma 4.12 we obtain

$$
\int_{2}^{x} \frac{d Q(t)}{\log t}=O\left(x^{\frac{d}{2}}\right) .
$$

For $P(x)$, the integration by parts leads to

$$
\int_{2}^{x} \frac{d P(t)}{\log t}=\frac{P(x)}{\log x}+\sum_{i=1}^{d} \frac{P_{i}(x)}{x^{i}(\log x)^{2}}+\int_{2}^{x} \frac{P_{d}(t)}{t^{d+1}(\log t)^{2}} d t+O(1),
$$

where the definition of $P_{i}(x)$ is (4.8). Proposition 3.1 implies

$$
\int_{0}^{R} \frac{1}{x^{d+1}} d N_{\Gamma}(x)=\log R
$$

which leads us to

$$
\sum_{0<t_{n} \leq R} \frac{1}{\left|s_{n}\right|^{d+1}}+\sum_{0<t_{n} \leq R} \frac{1}{\left|\tilde{s}_{n}\right|^{d+1}}=O(\log R) .
$$

Considering (4.11) with the above estimate (4.29), we obtain

$$
P_{d}(x)=O\left(x^{\frac{3}{2} d} \log x\right) .
$$


This gives

$$
P_{i}(x)=O\left(x^{\frac{d}{2}+i} \log x\right) .
$$

By substituting these estimates into (4.28), we have

$$
\int_{2}^{x} \frac{d P(t)}{\log t}=\frac{P(x)}{\log x}+O\left(\frac{x^{\frac{d}{2}}}{\log x}\right) .
$$

Applying the estimates (4.26), (4.27) and (4.30) to (4.25) gives the relation between $\pi_{\Gamma}(x)$ and $P(x)$ :

$$
\pi_{\Gamma}(x)=\sum_{n=0}^{M} \operatorname{li}\left(x^{s_{n}}\right)+\sum_{n=0}^{M} \operatorname{li}\left(x^{\tilde{s}_{n}}\right)+\frac{P(x)}{\log x}+O\left(\frac{x^{\frac{d}{2}}}{\log x}\right)+O\left(x^{\frac{d}{2}}\right) .
$$

By Theorem 4.11, we reach our main theorem, Theorem 1.2 .

\section{REMARK}

The necessary ingredient in the proof of the essential cuspidality which is our assumption (1.4) of Theorem 1.2, is an estimate of the determinant of the scattering matrix, which comes from the constant terms of the Eisenstein series for $\Gamma$. In this section, we introduce Reznikov's paper which indicates the essentially cuspidality for rank one Lie groups. It is sufficient to prove that the constant term of these Eisenstein series is a ratio of functions of order one. For this problem, Reznikov ([9]) prove the following theorem.

Theorem 5.1. The determinant of the Eisenstein matrix for a congruence subgroup of the unit group of a split rational quadratic form in $S O(d+1,1)$ is a ratio of function of order one.

\section{ACKNOWLEDGMENT}

I would like to express my sincere gratitude to Professor S. Koyama for suggesting this problem and for his great encouragement. This work was supported by the JSPS Research Fellowship for Young Scientists.

\section{REFERENCES}

[1] R. Gangolli, The length spectra of some compact manifolds of negative curvature, J. Differential Geometry 12 (1977), pp. 403-424. MR0650997 (58:31311)

[2] R. Gangolli and G. Warner, Zeta functions of Selberg's type for some non-compact quotients of symmetric spaces of rank one, Nagoya Math. J. 78 (1980), pp. 1-44. MR0571435|(82m:58049)

[3] Harish-Chandra, Spherical functions on a semi-simple Lie group (I, II), Amer. J. Math. 80 (1958), pp. 241-310, 533-613. MR0094407(20:925) MR0101279(21:92)

[4] D. Hejhal, The Selberg trace formula for PSL $(2, r) I$, Lect. Notes Math. 548, Springer, Berlin Heidelberg, New York (1976). MR0439755 (55:12641)

[5] A. E. Ingham, The distribution of prime numbers, Cambridge Univ. Press (1932). MR1074573 (91f:11064) (Reprint)

[6] M. Nakasuji, Prime geodesic theorem via the explicit formula of $\Psi$ for hyperbolic 3-manifolds, Proceedings Japan academy 77A (2001), pp. 130-133. MR1857290 (2002j:11099)

[7] M. Nakasuji, Prime geodesic theorem for hyperbolic 3-manifolds: general cofinite cases, $F_{O}$ rum Math. 16 (2004), no. 3, pp. 317-363. MR2050187 (2005g:11085)

[8] A. Postnikov, Tauberian theory and its applications, Proc. Steklov Inst. Math. Issue 2 (1980). MR0603991 (82f:40012b) 
[9] A. Reznikov, Eisenstein matrix and existence of cusp forms in rank one symmetric spaces, Geom. Funct. Anal. 3 no. 1 (1993). MR1204788 (94d:11034)

[10] G. Warner, Selberg's trace formula for non-uniform lattices: The R-rank one case, Advances in Math. Studies 6 (1979), pp. 1-142. MR0535763(81f:10044)

Department of Mathematics, Keio University, 3-14-1 Hiyoshi, 223-8522, Japan

E-mail address: nakasuji@math.keio.ac.jp 